\title{
Next-Generation Sequencing (NGS) in the Prediction of Pancreatic Malignancy: Does Cell Free Mean Error Free?
}

\author{
V. V. Ravikanth ${ }^{1}$
}

Published online: 27 March 2020

(c) Springer Science+Business Media, LLC, part of Springer Nature 2020

The prevalence of pancreatic cysts incidentally detected in the general population is reportedly high $(\sim 49.1 \%)$ with their number and size increasing with age [1]. Pancreatic cysts commonly encountered in clinical practice include serous cystadenomas (SCAs), mucinous cystic neoplasms (MCNs), and intraductal papillary mucinous neoplasm (IPMNs). The management of the pancreatic cysts usually depends on their type; SCAs with a low malignant potential require minimal or no surveillance in the absence of symptoms. Surgical resection is recommended for low-grade neoplasms including solid-pseudopapillary neoplasms (SPN). Although follow-up is recommended by a few groups for asymptomatic MCNs, surgeons favor resection since these cysts have malignant potential, requiring long periods of close surveillance. Surgery is recommended for IPMNs involving the main pancreatic duct due to a greater risk of malignant transformation. In contrast, there is a much lower risk of malignancy in branch duct-type IPMNs where there is no involvement of the main duct; close surveillance is usually recommended, with surgery advised only in the presence of certain high-risk criteria [2].

Based on the above recommendations, a key clinical question is to ascertain whether a cyst is benign, and if so, it is important to determine its malignant potential that will inform whether surveillance or surgical resection is the most appropriate. Given these uncertainties, there is a demand for efficient prognostic biomarkers. Currently, this determination is based on clinical history and imaging studies such as MRI (magnetic resonance imaging), CT (computed tomography), and EUS (endoscopic ultrasonography). EUS coupled with EUS-guided fine needle aspiration enables the detailed visualization of the cyst with an additional benefit

V. V. Ravikanth

ravikanth.aig@gmail.com

1 Asian Healthcare Foundation (A Research Wing of Asian Institute of Gastroenterology), 6-3-661, Somajiguda, Hyderabad, Telangana 500082, India of obtaining aspirate cytology and biochemical and molecular testing that increases the diagnostic and predictive accuracy of the evolution of pancreatic cysts.

Although carcinoembryonic antigen (CEA) is often used as an accurate marker to differentiate mucinous from nonmucinous cysts (IPMNs and MCNs from others), recent studies have raised questions regarding optimal cutoffs and the accuracy of CEA in this determination. Although elevated cyst fluid amylase levels exclude a pseudocyst from other types of pancreatic cysts with greater specificity (98\%), they do not differentiate IPMNs from MCNs. Other biochemical tumor markers including CA 72-4, CA 125, CA 19-9, and CA 15-3 have diagnostic accuracy inferior to CEA and therefore are not used in clinical practice [3]. Whole exome analysis employing next-generation sequencers (NGS) by Bert Vogelstein et al. of the genome from cysts including SCA, SPN, MCNs, and IPMNs identified that each cyst type had a distinct mutational profile. While a mutation in von Hippel-Lindau (VHL) gene was seen in SCAs, mutation in the CTNNBI gene was seen in SPNs. Mutations in $K R A S$ and RNF43 were seen in both IPMNs and MCNs. A mutation unique to IPMNs that was not seen in other cyst types was identified in the guanine nucleotide binding protein, alpha stimulating activity polypeptide $1(G N A S)$ gene. Apart from the mutations at single nucleotide level, $\mathrm{LOH}$ and aneuploidy were also assessed. These molecular markers have the ability to not only identify the cyst type but also the presence of high-grade dysplasia or invasive carcinoma in IPMNs which is attributed to the presence of mutations in SMAD4, TP53, loss of heterozygosity ( $\mathrm{LOH}$ ) chromosome 17 (ring finger protein $[R N F] 43$ or TP53 loci), or aneuploidy in chromosomes 5p, 8p, 13q, 18q. Ongoing research efforts are underway to identify reliable markers that can help determine the malignant potential of pancreatic cysts.

In this issue of Digestive Diseases and Sciences, Paziewska et al. [4] studied the pancreatic cyst fluid in order to exploit potential biomarkers by identifying variants in the cell-free DNA (cfDNA) shed into the cavity of pancreatic 
cysts. They sequenced cfDNA obtained from 71 patients who underwent EUS-FNA on the Ion proton platform using the comprehensive cancer panel. Moreover, they also determined the KRAS and GNAS mutations on the digital PCR platform. The group demonstrated relatively higher rates of somatic mutations of cancer-related genes including KRAS and GNAS in the cfDNA isolated from pancreatic cyst fluids irrespective of the cyst type.

The key to predict early malignancy accurately in cfDNA involves: (a) efficient isolation of cfDNA, (b) sequencing to identify the variants that include a useful predictive marker or panel of markers, and (c) platform(s) and pipeline(s) that can identify a few variant alleles from the tumor in the background of abundant host DNA.

Cell death is the major origin of cfDNA as evidenced by studies evaluating its properties. cfDNA is inherently degraded, double stranded, and averages $150 \mathrm{bp}$ in length. Although studies have reported that there is an increased integrity of cfDNA in patients with cancer as compared to neoplasm-free controls, there are other conflicting reports that suggest decreased cfDNA integrity correlated with inferior outcome. The amount of cfDNA shed into the pancreatic cyst fluid might vary widely between patients due to largely unknown factors. Collecting and processing the cyst fluid is the other major determinant of how well the cfDNA can be stabilized and isolated for further processing including amplification and sequencing. Most of the understanding of the biology of cfDNA and the optimal methods for collection and extraction are derived from the study of fetal cfDNA. Extensive experiments on fetal and maternal DNA have identified factors that affect its stability, yield, and source of contamination. Most of the studies of cfDNA now emphasize the importance of standardization, starting with collection of blood or other material, through processing and DNA extraction in order ensure that data from individual studies remain closely comparable.

Efficient amplification of the targets from cfDNA templates is expected with an amplicon size of $\sim 150 \mathrm{bp}$, corresponding to the median size of the cfDNA [5], a finding supported by an earlier study that reported an increase (5-20-fold) in the percentage of mutant molecules of the adenomatous polyposis coli $(A P C)$ gene when the template size was decreased from 1296 to 100 bp [6]. Furthermore, a recent study identified that apart from variants $(K R A S$ and TP53), copy number alterations (CNAs-17p, 9p, ARIDIA, $18 \mathrm{q}, 9 \mathrm{p} 213, S M A D 4)$ are preferentially altered early in the tumorigenesis process [7]. This suggests that for effective identification of early tumorigenesis, it is not only the variants that have to genotyped but CNAs also have to be assessed.

The mutant allele frequency in the cfDNA of a confirmed cancer patient can be as low as $0.01 \%$ [8]. Since this frequency would be much lower in the cfDNA from the pancreatic cyst fluid, any analysis pipeline that can ignore the false negatives improves diagnostic accuracy. Nevertheless, as noted by important studies including that by Guntry et al. [9], the significant limitation of the current massively parallel sequencing technologies is its inefficiency in identifying low-abundance mutations (somatic variants) that are involved in the early neoplastic transformation, an argument that is generally based on the comparison between natural mutation rates in mammalian cells and error rates of the sequencing platforms.

The other issue cofounding the prediction of tumorigenesis is that it is not only single nucleotide variants that are involved, but also copy number variants and variability in the methylation of promoter sequences, that may contribute to early neoplastic transformation. Although the ability to sequence templates employing massively parallel sequencing has grown immensely over the years, the understanding of the genome and the contribution and combination of the events leading to the early neoplastic transformation has not matched the progress of sequencing technologies. Furthermore, most of the research that has studied the process of tumorigenesis usually include advanced-stage tumors since it is technically challenging to include samples from early stages which in turn has limited the understanding of the early factors driving the tumorigenesis process and the variants that actually initiate the tumors, the so called driver variant(s).

In conclusion, standard and robust protocols starting from collection of the testing material (plasma, pancreatic cyst fluid) to primer designing and data analysis pipelines have to be developed. Robust molecular markers or a combination of markers such as CNAs and methylation that have the potential to predict malignant potential have to be identified. Powerful analysis tools that have the ability to detect variants in the background of host DNA with minimal to no false positives have to be developed. With these novel and powerful methods, it is hoped that all pancreatic cystic lesions can be treated appropriately, with minimal missed tumors on the one hand and minimal unnecessary surgeries on the other.

\section{References}

1. Kromrey ML, Bülow R, Hübner J, et al. Prospective study on the incidence, prevalence and 5-year pancreatic-related mortality of pancreatic cysts in a population-based study. Gut. 2018;67:138-145.

2. Ngamruengphong S, Lennon AM. Analysis of pancreatic cyst fluid. Surg Pathol Clin. 2016;9:677-684.

3. Brugge WR, Lewandrowski K, Lee-Lewandrowski E, et al. Diagnosis of pancreatic cystic neoplasms: a report of the cooperative pancreatic cyst study. Gastroenterology. 2004;126:1330-1336. 
4. Paziewska A, Polkowski M, Goryca K et al. Mutational mosaics of cell-free DNA from pancreatic cyst fluids. Dig Dis Sci. (Epub ahead of print). https://doi.org/10.1007/s10620-019-06043-1.

5. Kornberg RD, Lorch Y. Twenty-five years of the nucleosome, fundamental particle of the eukaryote chromosome. Cell. 1999;98:285-294.

6. Diehl F, Li M, Dressman D, et al. Detection and quantification of mutations in the plasma of patients with colorectal tumours. Proc Natl Acad Sci USA. 2005;102:16368-16373.

7. Gerstung M, Jolly C, Leshchiner I, Dentro S, et al. The evolutionary history of 2,658 cancers. Nature. 2020;578:122-128.

8. Volik S, Alcaide M, Morin RD, et al. Cell-free DNA (cfDNA): clinical significance and utility in cancer shaped by emerging technologies. Mol Cancer Res. 2016;14:898-908.
9. Gundry M, Vijg J. Direct mutation analysis by high-throughput sequencing: from germline to low-abundant, somatic variants. Mutat Res. 2012;729:1-15.

Publisher's Note Springer Nature remains neutral with regard to jurisdictional claims in published maps and institutional affiliations. 